\begin{tabular}{|c|c|}
\hline Title & Reflection and transmission of light in multilayers perturbed by picosecond strain pulse propagation \\
\hline Author(s) & Matsuda, O.; Wright, O. B. \\
\hline Citation & $\begin{array}{l}\text { Journal of the Optical Society of A merica B: Optical Physics, 19(12), 3028-3041 } \\
\text { https://doi.org/10.1364/JOSA B.19.003028 }\end{array}$ \\
\hline Issue Date & 2002-12-02 \\
\hline Doc URL & http:/hdl.handle.net/2115/44497 \\
\hline Rights & () 2002 Optical Society of A merica \\
\hline Type & article \\
\hline File Information & JOSA B19-12_3028-3041.pdf \\
\hline
\end{tabular}

Instructions for use 


\title{
Reflection and transmission of light in multilayers perturbed by picosecond strain pulse propagation
}

\author{
O. Matsuda* and O. B. Wright \\ Department of Applied Physics, Faculty of Engineering, Hokkaido University, Sapporo 060-8628, Japan
}

Received December 21, 2001; revised manuscript received May 31, 2002

\begin{abstract}
We derive analytical formulas for the modulation of the reflectance and transmittance of light normally incident on a multilayer thin-film structure whose refractive indices are perturbed by an ultrashort optical pulse. The formulas, expressed in compact form, should prove useful for analysis of a wide range of ultrashort timescale experiments on multilayers as well as longer time-scale photoacoustic and photothermal experiments based on optical probing. We demonstrate our method by the analysis of the modulated reflectance variation of a $\mathrm{SiO}_{2} / \mathrm{Cr}$ structure in which picosecond acoustic pulses have been optically excited. (C) 2002 Optical Society of America
\end{abstract}

OCIS codes: $320.7120,230.4170,300.6430,120.4290,310.6860,310.6870$.

\section{INTRODUCTION}

The reflection of light from multilayer thin-film structures is an important problem for a variety of applications. Recently such structures with layer thicknesses in the submicrometer range have been the focus of a wide range of ultrashort time-scale experiments that probe the inhomogeneous modulation of the dielectric constant along the stacking direction induced by an incident ultrashort optical pulse. ${ }^{1-17}$ This modulation can be caused by, for example, the diffusion and relaxation of nonequilibrium electron and hole distributions, thermal diffusion, or coherent acoustic-phonon propagation. The main aim of these experiments is to probe such phenomena and extract quantitative information about the relevant spatiotemporally varying field. Another popular aim is to measure the electronic, thermal, or mechanical properties of the multilayer film structure itself.

To interpret the experimental results for multilayers in the general case, one needs to handle the problem of light scattering by multilayer films with inhomogeneously modulated optical constants and thicknesses. This procedure becomes extremely complex for transparent or partially transparent multilayer films because optical scattering from all or part of the sample makes a contribution to the final amplitude and phase of the reflected or transmitted light wave. Many commercially and physically important samples such as semiconductor heterostructures or multilayer metal stacks are partially transparent at visible or near-visible optical wavelengths when film thicknesses are in the nanometer to micrometer range.

For one- or two-layer structures, techniques have been developed to deal with the problem of multiple optical reflections when one is calculating the modulation in reflectance or transmittance. ${ }^{14-19}$ For samples with more layers, however, the problem becomes extremely complex and difficult to handle. ${ }^{20-23}$ Therefore there is a need for a simpler approach to analyze the modulation in the reflectance or transmittance of a multilayer sample with an arbitrary number of layers subject to perturbations in the dielectric constant or layer thickness.

In this paper we describe, for what to our knowledge is the first time, the derivation of such a generalized analytical theory. The reflectance and transmittance changes are expressed in a simple ready-to-use form that has not been given in previous treatments. ${ }^{20-23}$ The theory can be used to analyze ultrashort time-scale optical pump and probe experiments on arbitrary multilayer structures with normal probe light incidence when the changes induced in the dielectric constants or the layer thicknesses of the structure are relatively small compared to their equilibrium values. It is applicable, for example, to the interpretation of subpicosecond transient reflectance or transmittance in multilayers with spatiotemporally varying electron distributions that can be described by a suitable dynamical model and to the analysis of data from multilayers involving picosecond thermoreflectance or picosecond acoustics. The main focus of the present paper is on the application to picosecond acoustics. It should also be possible to apply the method to longer time-scale photothermal and photoacoustic experiments on multilayers that make use of optical probing. To demonstrate the practical application of the theory, we describe ultrafast optical measurements on a double-layer $\mathrm{SiO}_{2}$-Cr thin-film structure on a fused-silica substrate involving the propagation of picosecond acoustic pulses and show how the results can be used to extract information about the film parameters.

In Subsection 2.A we consider how the optical reflectance and transmittance are affected by spatially dependent perturbations-such as those arising from strain or temperature variations-in the multilayer structure. We consider the particular example of an optically excited strain distribution in a multilayer by means of a simpli- 
fied model of strain generation and propagation in Subsection 2.B. The experimental procedure is described in Section 3, and the experimental data are analyzed in Section 4 on the basis of the theoretical model. In Appendixes A-C the details of the light-scattering theory, including the derivation of the Green's function, are given.

\section{THEORY}

A. Light Scattering by an Inhomogeneous Multilayer

1. Theoretical Background

The typical dimensions of the multilayers considered in this paper range from the order of a nanometer to approximately a micrometer, and experimental time scales of concern here are of picosecond order. Photoexcitation of such a sample by ultrashort optical pulses causes perturbations, in the guise of strain, ${ }^{1-7,13-16}$ temperature, ${ }^{9-13,23-27}$ and excited carrier distributions, ${ }^{10-13,24-29}$ all of which are varying in both space and time. The effect of these perturbations on the dielectric constants has been extensively investigated since the advent of ultrashort-pulse lasers, thanks to the ease of monitoring the variation in the optical reflectance or transmittance with the optical pump and probe technique. It is less well known that a propagating strain distribution in a sample can also significantly affect the optical reflectance or transmittance through the induced motion of the sample interfaces on ultrashort time scales, independent of the coupling of the strain to the dielectric constants. ${ }^{2,14,15}$ The overall optical reflectance, transmittance, and phase in a multilayer sample can therefore vary with time after optical excitation according to the following two mechanisms: (1) perturbations in the dielectric constants and (2) perturbations in the interface positions (implying variations in the film thicknesses). In the present treatment we consider optical pulses with a coherence length that is much greater than the total thickness of the transparent (or partially transparent) portion of the multilayer in question. The multiple optical reflection of the light can then be treated as if the light were monochromatic. With 100-fs Fourier-transformlimited optical pulses, the corresponding coherence length is $\sim 30 \mu \mathrm{m}$. Multilayers thinner than $\sim 5-10 \mu \mathrm{m}$ can be treated with reasonable accuracy with the present theory in this case.

It is not uncommon in experiment to monitor either the reflectance and transmittance variations or both by use of delayed probe light pulses, making use of the optical pump and probe technique. The technique of monitoring both reflectance and transmittance variations allows more information about the sample response to be derived. For example, the separation of the variations of the real and complex parts of the dielectric constant can be achieved for a sample consisting of a partially transparent layer on a substrate..$^{9,11}$ It is also possible to simultaneously detect both intensity and phase changes in reflection, also providing more information than an intensity measurement alone. ${ }^{2-4}$ This can be done, for example, with an interferometric or beam deflection setup. The theoretical approach presented in this paper also al- lows the extension of the analysis of this kind of experiment to more complex multilayer geometries.

Because we compare the theory with experimental results in the field of laser picosecond acoustics, in this paper we mainly focus on the effect of induced strain distributions, although the equations obtained apply equally well to other distributions provided they can be expressed in terms of perturbations in the linear dielectric constants. Laser picosecond acoustics is based on the optical excitation of a picosecond acoustic-phonon pulse in a sample and the optical detection of the phonon propagation through the variation in reflectance, transmittance, or phase of a probe light pulse., ${ }^{1,2}$ Although the technique has been applied successfully to opaque multilayer structures with several layers, the case of partially transparent or transparent multilayers is less well understood because of the increased complexity of the analysis. In spite of the continuing interest shown in the application to superlattices, ${ }^{5-8}$ only the case of a single transparent or partially transparent layer on another opaque layer has been properly analyzed to include interface motion as well as variations in the dielectric constants. ${ }^{14-16}$ The exact treatment for the general case of more than one transparent or partially transparent layer presented here should therefore be extremely valuable in the field of laser picosecond acoustics for application to samples of arbitrary multilayer structure. The method should also be useful in the field of femtosecond probing of semiconductor heterostructures. In addition, it should be applicable to more conventional experiments, based on photothermal or photoacoustic effects, that involve optical probing in transparent or partially transparent multilayers. ${ }^{30-33}$

\section{Solution of the Inhomogeneous Wave Equation}

Here we aim to calculate the complex reflectance or transmittance change for coherent light normally incident on a multilayer sample. Each layer is assumed to be optically isotropic in its equilibrium state, that is, at zero strain, with no excited carriers and at constant temperature. In addition, each layer is also assumed to be mechanically, thermally, and electronically isotropic. The $z$ axis is defined along the stacking direction of the multilayer, as shown in Fig. 1. Each interface and the surface are

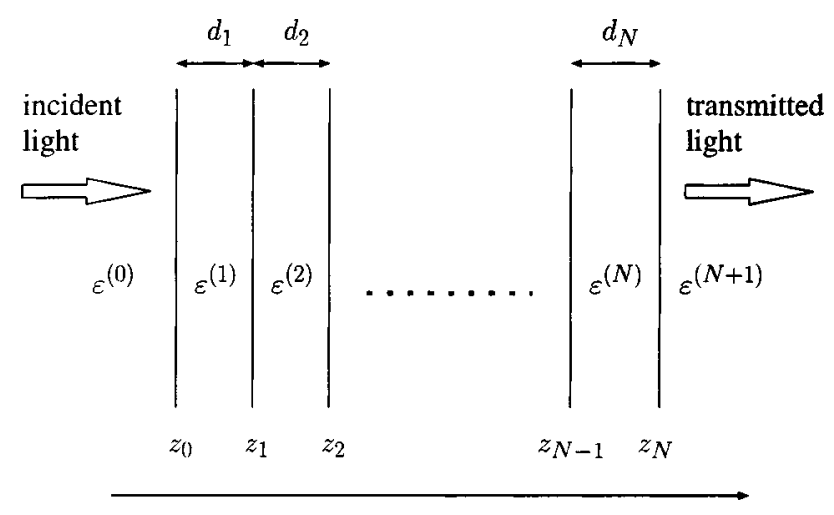

Fig. 1. $N$ layers on a substrate. The incident light comes from $z<0$. Each layer is assumed to be optically isotropic in its equilibrium state. 
therefore perpendicular to the $z$ axis. The incident light comes from $z<0$ and propagates along the positive $z$ direction.

The lateral extent of the irradiated region is assumed to be much larger than the scale of the depth under consideration. For example, a typical laser beam spot with a radius of $10 \mu \mathrm{m}$ would be appropriate for a multilayer film for which the optical penetration depth is of a submicrometer order. In this case it can be assumed that the strain, temperature, and excited carrier distributions in the sample are dependent only on the $z$ coordinate and on the time $t$.

The general wave equation derived from the Maxwell's equations is

$$
\left(\nabla^{2}-\operatorname{grad} \operatorname{div}\right) \mathbf{E}(\mathbf{x}, t)=\mu_{0} \frac{\partial^{2}}{\partial t^{2}} \mathbf{D}(\mathbf{x}, t) .
$$

By considering monochromatic light with angular frequency $\omega$, we can omit the common time-dependent factor $\exp (-i \omega t)$. Under a linear approximation, the electric displacement $\mathbf{D}$ is given as $\mathbf{D}=\epsilon_{0} \widetilde{\boldsymbol{\epsilon}}(\omega) \mathbf{E}$, with dielectric tensor $\widetilde{\epsilon}$; and the wave equation of Eq. (1) is reduced to

$$
\left(\nabla^{2}-\operatorname{grad} \operatorname{div}+k^{2} \widetilde{\boldsymbol{\epsilon}}\right) \mathbf{E}(\mathbf{x})=0,
$$

where $k=\sqrt{\mu_{0} \epsilon_{0}} \omega$ is the wave vector of light in vacuum. Because the characteristic time scale of concern here for strain propagation, thermal diffusion, excited carrier relaxation, or carrier diffusion is much longer than the period of the light wave, we can treat the dielectric tensor as a quasi-static quantity. We therefore neglected terms related to the time derivatives of $\widetilde{\epsilon}$. However, the spatial dependence of $\tilde{\epsilon}$ is retained.

As the scattering and reflection of light are governed by the spatial variation of the dielectric tensor that depends only on the $z$ coordinate here, no changes in the $x$ and $y$ components of the light wave vector are expected for any angle of incidence. In our case of normal incidence, it is possible to write $\mathbf{E}(\mathbf{x})=\mathbf{E}(z)$. Then the wave equation of Eq. (2) is reduced to

$$
\left\{\left[\begin{array}{ccc}
\partial^{2} / \partial z^{2} & 0 & 0 \\
0 & \partial^{2} / \partial z^{2} & 0 \\
0 & 0 & 0
\end{array}\right]+k^{2} \widetilde{\boldsymbol{\epsilon}}\right\} \mathbf{E}(z)=0 .
$$

Because no off-diagonal component in $\widetilde{\epsilon}$ and $\epsilon_{x x}=\epsilon_{y y}$ is expected here, as shown in Appendixes A and B, Eq. (3) includes two independent and equivalent wave equations for $E_{x}(z)$ and $E_{y}(z)$, and, in addition, $E_{z}(z)=0$. The light wave is purely transverse, and there is no mixing between $x$ and $y$ polarizations. Without loss of generality, we consider only linearly polarized incident light and take the $x$ axis along the direction of polarization.

The dielectric tensor component of interest, $\epsilon \equiv \epsilon_{x x}$ $=\epsilon_{y y}$, can be divided into homogeneous and inhomogeneous parts, $\epsilon_{h}(z)$ and $\epsilon_{i h}(z)$. The material-dependent dielectric constant in the equilibrium state forms the homogeneous part. It is a staircaselike function and has a constant value within each layer characterized by the label $n(0 \leqslant n \leqslant N+1)$ :

$$
\epsilon_{h}(z)=\epsilon^{(n)} \text { for } z_{n-1}<z<z_{n} .
$$

Special values of $z_{n}$ are $z_{-1}=-\infty$ and $z_{N+1}=+\infty$.
The inhomogeneous part $\epsilon_{i h}$ is the sum of bulk and interface contributions, $\epsilon_{b}$ and $\epsilon_{i f}$, respectively. In this paper, the strain-induced bulk contribution $\epsilon_{b}$ is given by

$$
\epsilon_{b}(z)=P_{12}^{(n)} \eta(z) \quad \text { for } z_{n-1}<z<z_{n},
$$

where $P_{12}^{(n)}$ is the photoelastic constant of the $n$th layer and $\eta(z) \equiv \eta_{z z}(z)$ is the $z z$ tensor component of the strain distribution (Appendix A). The interface contribution $\epsilon_{i f}$ is caused by the displacement of the interface because of the strain and is given by

$$
\begin{aligned}
& \epsilon_{i f}(z) \\
& \quad= \begin{cases}\epsilon^{(n)}-\epsilon^{(n+1)} & \text { for } u\left(z_{n}\right)>0, z_{n}<z<z_{n}+u\left(z_{n}\right) \\
\epsilon^{(n+1)}-\epsilon^{(n)} & \text { for } u\left(z_{n}\right)<0, z_{n}+u\left(z_{n}\right)<z<z_{n}, \\
0 & \text { otherwise, }\end{cases}
\end{aligned}
$$

where

$$
u(z)=-\int_{z}^{\infty} \eta\left(z^{\prime}\right) \mathrm{d} z^{\prime}
$$

is the displacement in the $+z$ direction.

We proceed by assuming that the solution for the homogeneous wave equation, defined over all space, is known:

$$
\left[\frac{\partial^{2}}{\partial z^{2}}+k^{2} \epsilon_{h}(z)\right] E_{0}(z)=0,
$$

and likewise for the Green's function defined by the equation

$$
\left[\frac{\partial^{2}}{\partial z^{2}}+k^{2} \epsilon_{h}(z)\right] G\left(z, z^{\prime}\right)=-\delta\left(z-z^{\prime}\right) .
$$

The derivations of the homogeneous solution $E_{0}(z)$ and the Green's function $G\left(z, z^{\prime}\right)$ are given in Appendix C. Physically, $G\left(z, z^{\prime}\right)$ represents the response at $z$ caused by a localized disturbance at $z^{\prime}$. Both $E_{0}(z)$ and $G\left(z, z^{\prime}\right)$ should satisfy the same boundary conditions at each interface. By using these solutions, we can express the solution to the inhomogeneous wave equation in Eq. (3) as

$$
\begin{aligned}
E(z)= & E_{0}(z)+\int_{-\infty}^{\infty} k^{2} \epsilon_{i h}\left(z^{\prime}\right) E\left(z^{\prime}\right) G\left(z, z^{\prime}\right) \mathrm{d} z^{\prime} \\
\simeq & E_{0}(z)+\int_{-\infty}^{\infty} k^{2} \epsilon_{i h}\left(z^{\prime}\right) E_{0}\left(z^{\prime}\right) G\left(z, z^{\prime}\right) \mathrm{d} z^{\prime} \\
& +\int k^{4} \epsilon_{i h}\left(z^{\prime}\right) \epsilon_{i h}\left(z^{\prime \prime}\right) E_{0}\left(z^{\prime \prime}\right) G\left(z, z^{\prime}\right) \\
& \times G\left(z^{\prime}, z^{\prime \prime}\right) \mathrm{d} z^{\prime} \mathrm{d} z^{\prime \prime}+\cdots .
\end{aligned}
$$

As described in Appendix C, the solutions $E_{0}(z)$ and $E(z)$ each contain two components propagating in the $+z$ and $-z$ directions. Because we consider the reflection and transmission of light coming from $z<0$, the $-z$ propagating components of $E_{0}(z)$ and $E(z)$ in the region $z$ $=+\infty$ should be zero. The boundary conditions for $E(z)$ at $z= \pm \infty$ require that the $+z$ propagating component of $G\left(-\infty, z^{\prime}\right)$ and the $-z$ component of $G\left(+\infty, z^{\prime}\right)$ should both be zero. This formalism is exactly equivalent to 
that for particle scattering by an arbitrary static potential in quantum mechanics. When $\epsilon_{i h}$ is small, expansion up to first order in Eq. (9) is sufficient in many experiments. It is also possible to include higher-order terms in the expansion for large perturbations.

The solution to Eq. (7) is constructed from the solutions for each layer where we consider the boundary conditions at each interface. This homogeneous solution for a multilayer structure can be derived by a transfer matrix technique, as is well known. ${ }^{34,35}$ We give the derivation in Appendix C. The solution for the $n$th layer has the form

$$
a_{n} \exp \left(i k_{n} \zeta_{n}\right)+b_{n} \exp \left(-i k_{n} \zeta_{n}\right),
$$

where $a_{n}$ and $b_{n}$ are constant electric fields that satisfy the given boundary conditions, and $\zeta_{n}$ and $k_{n}$ are defined as follows:

$$
\begin{gathered}
\zeta_{n} \equiv\left\{\begin{array}{cc}
z-z_{n-1} & \text { for } n \geqslant 1, \\
z & \text { for } n=0,
\end{array}\right. \\
k_{n} \equiv \sqrt{\epsilon^{(n)}} k \equiv\left(N_{n}+i K_{n}\right) k,
\end{gathered}
$$

where $N_{n}+i K_{n}$ is the complex refractive index and $k_{n}$ is the complex wave number in medium $n$.

The Green's function necessary for the reflectivity calculation is given as

$$
\begin{aligned}
& G\left(z, z^{\prime}\right)=\frac{i}{2 k_{0} a_{0}} \exp \left(-i k_{0} z\right) E_{0}\left(z^{\prime}\right) \\
& \quad \text { for } z^{\prime}>z, z<0 .
\end{aligned}
$$

Incorporating Eqs. (4), (5), and (11) in Eq. (9), we obtain the following result at $z<0$ beyond the perturbed region:

$$
\begin{aligned}
E(z) \simeq & E_{0}(z)+\int_{z}^{+\infty} k^{2}\left[\epsilon_{b}\left(z^{\prime}\right)\right. \\
& \left.+\epsilon_{i f}\left(z^{\prime}\right)\right] E_{0}\left(z^{\prime}\right) G\left(z, z^{\prime}\right) \mathrm{d} z^{\prime} \\
= & E_{0}(z)+\int_{z}^{+\infty} k^{2} \epsilon_{b}\left(z^{\prime}\right) E_{0}\left(z^{\prime}\right) G\left(z, z^{\prime}\right) \mathrm{d} z^{\prime} \\
& +\sum_{n=0}^{N} \int_{z_{n}}^{z_{n}+u\left(z_{n}\right)} k^{2}\left[\epsilon^{(n)}-\epsilon^{(n+1)}\right] E_{0}\left(z^{\prime}\right) \\
& \times G\left(z, z^{\prime}\right) \mathrm{d} z^{\prime} \\
= & a_{0} \exp \left(i k_{0} z\right)+b_{0} \exp \left(-i k_{0} z\right) \\
& +\frac{i k^{2} \exp \left(-i k_{0} z\right)}{2 k_{0} a_{0}}\left\{\int _ { z } ^ { 0 } P _ { 1 2 } ^ { ( 0 ) } \eta ( z ^ { \prime } ) \left[a_{0} \exp \left(i k_{0} z^{\prime}\right)\right.\right. \\
& \left.+b_{0} \exp \left(-i k_{0} z^{\prime}\right)\right]^{2} \mathrm{~d} z^{\prime}+\sum_{n=1}^{N+1} \int_{0}^{d_{n}} P_{12}^{(n)} \eta\left(z^{\prime}\right. \\
& \left.+z_{n-1}\right)\left[a_{n} \exp \left(i k_{n} z^{\prime}\right)+b_{n} \exp \left(-i k_{n} z^{\prime}\right)\right]^{2} \mathrm{~d} z^{\prime} \\
& \left.+\sum_{n=1}\left(a_{n}+b_{n}\right)^{2}\left[\epsilon^{(n-1)}-\epsilon^{(n)}\right] u\left(z_{n-1}\right)\right\}, \quad(12) \\
& \left.+\sum_{n}\right)
\end{aligned}
$$

where $d_{N+1}=+\infty$. Thus the relative change in the complex reflectance is given by

$$
\begin{aligned}
\frac{\delta r}{r}= & \frac{i k^{2}}{2 k_{0} a_{0} b_{0}}\left\{\int _ { z } ^ { 0 } P _ { 1 2 } ^ { ( 0 ) } \eta ( z ^ { \prime } ) \left[a_{0} \exp \left(i k_{0} z^{\prime}\right)\right.\right. \\
& \left.+b_{0} \exp \left(-i k_{0} z^{\prime}\right)\right]^{2} \mathrm{~d} z^{\prime}+\sum_{n=1}^{N+1} \int_{0}^{d_{n}} P_{12}^{(n)} \eta\left(z^{\prime}\right. \\
& \left.+z_{n-1}\right)\left[a_{n} \exp \left(i k_{n} z^{\prime}\right)+b_{n} \exp \left(-i k_{n} z^{\prime}\right)\right]^{2} \mathrm{~d} z^{\prime} \\
& \left.+\sum_{n=1}^{N+1}\left(a_{n}+b_{n}\right)^{2}\left[\epsilon^{(n-1)}-\epsilon^{(n)}\right] u\left(z_{n-1}\right)\right\},
\end{aligned}
$$

where $r \equiv b_{0} / a_{0}$ is the complex reflectance for the unperturbed multilayer. The first term in Eq. (13) accounts for the possible modulation of the transparent medium in front of the multilayer, the second term accounts for the bulk photoelastic effect, and the third term accounts for the interface motion. The result can be extended in a straightforward way to include the effects of the temperature variation $\Delta T$ and the excited electron density $\Delta n$. For this purpose, the quantity $P_{12} \eta$ in Eq. (13) should be replaced by $P_{12} \eta+s \Delta T+\gamma \Delta n$, where $s$ and $\gamma$ are constants [See Eq. (A1)].

We now describe some concrete examples for simple cases and make comparisons with related results previously reported in the literature. One of the simplest cases is a single semi-infinite isotropic material whose flat surface at $z=0$ is exposed to vacuum. The dielectric constants for each layer are $\epsilon^{(0)}=1, \epsilon^{(1)}=\epsilon$. Thus the wave vectors in each layer are $k_{0}=k=\omega / c$ and $k_{1}$ $=\sqrt{\epsilon} k$. In this case Eq. (13) reduces to

$$
\begin{aligned}
\frac{\delta r}{r} & =\frac{2 i k}{1-\epsilon} \int_{0}^{+\infty} \Delta \epsilon\left(z^{\prime}\right) \exp \left(2 i k_{1} z^{\prime}\right) \mathrm{d} z^{\prime}+2 i k u(0) \\
& =\frac{4 i k \tilde{n}}{1-\tilde{n}^{2}} \int_{0}^{+\infty} \Delta \widetilde{n}\left(z^{\prime}\right) \exp \left(2 i k \widetilde{n} z^{\prime}\right) \mathrm{d} z^{\prime}+2 i k u(0),
\end{aligned}
$$

where $\widetilde{n}+\Delta \widetilde{n} \simeq \sqrt{\epsilon+\Delta \epsilon}$ is the complex refractive index. This formula is equivalent to that previously reported. ${ }^{4}$ The expression for relative variation of the reflectivity $\Delta R / R=2 \operatorname{Re}(\delta r / r)$ agrees with previous research. $1,10,19$

Another example is the case of a transparent layer of dielectric constant $\epsilon_{1}$ of thickness $d$ on a substrate of dielectric constant $\epsilon_{2}$. The medium in front of the transparent layer is a vacuum. The relative reflectance for this case is given by

$$
\begin{aligned}
\frac{\delta r}{r}= & \frac{i k}{2 a_{0} b_{0}}\left\{\int _ { 0 } ^ { d } \Delta \epsilon ( z ^ { \prime } ) \left[a_{1} \exp \left(i k_{1} z^{\prime}\right)\right.\right. \\
& \left.+b_{1} \exp \left(-i k_{1} z^{\prime}\right)\right]^{2} \mathrm{~d} z^{\prime} \\
& +\int_{0}^{\infty} \Delta \epsilon\left(z^{\prime}+d\right) a_{2}^{2} \exp \left(2 i k_{2} z^{\prime}\right) \mathrm{d} z^{\prime} \\
& \left.+\left(a_{1}+b_{1}\right)^{2}\left(1-\epsilon_{1}\right) u(0)+a_{2}^{2}\left(\epsilon_{1}-\epsilon_{2}\right) u(d)\right\},
\end{aligned}
$$

with 


$$
\begin{aligned}
a_{0}= & \left(k+k_{1}\right)\left(k_{1}+k_{2}\right) \\
& +\left(k-k_{1}\right)\left(k_{1}-k_{2}\right) \exp \left(2 i k_{1} d\right), \\
b_{0}= & \left(k-k_{1}\right)\left(k_{1}+k_{2}\right) \\
& +\left(k+k_{1}\right)\left(k_{1}-k_{2}\right) \exp \left(2 i k_{1} d\right), \\
a_{1}= & 2 k\left(k_{1}+k_{2}\right), \\
b_{1}= & 2 k\left(k_{1}-k_{2}\right) \exp \left(2 i k_{1} d\right), \\
a_{2}= & 4 k k_{1} \exp \left(i k_{1} d\right) .
\end{aligned}
$$

This case with either real or complex $\epsilon_{1}$ has been treated previously exactly with a less compact formalism. ${ }^{14,16}$ Although the notation is different, Eq. (15) turns out to be identical to the result derived by Gusev. ${ }^{16}$ We also note that the second term in Eq. (13) agrees with the result quoted without proof by Perrin et al. ${ }^{3}$

We now turn to the case of the complex transmittance. Thin multilayers are often formed on a substrate with a thickness considerably greater than the multilayer itself. The reflection at the back surface of the substrate does not add coherently to the light emerging from the multilayer provided that the coherence length of the light pulse is short compared to the substrate thickness. This is the case for substrates with thickness $\gtrsim 30 \mu \mathrm{m}$ in the case of $100-f$ light pulses. For simplicity, we calculate only the electric field at a point $z>z_{N}$ that is beyond the perturbed region and within the $(N+1)$ th layer, that is, the substrate. For this calculation, we require $G\left(z, z^{\prime}\right)$ for $z>z_{N}$ in Eq. (9). To calculate this, we need to extend the range of application of the homogeneous solution of Eq. (10). So far, we have considered only the solution $E_{0}(z)$ for light coming from the $x<0$ region. In general, however, the $2 N+2$ constants $a_{n}, b_{n}$ in Eq. (10) are uniquely determined if any two of them are specified. A suitable symbolic representation of the solution for generalized $E_{0}$ is $E_{0}\left(z ; a_{i}{ }^{\prime}=a, b_{j}{ }^{\prime}=b\right)$, which means that $a_{i}{ }^{\prime}$ and $b_{j}{ }^{\prime}$ are specified. The primes are added to recall that the set $\left\{a_{n}{ }^{\prime}, b_{n}{ }^{\prime}\right\}$ is not necessarily the same as that used for the solution of $\mathbf{E}_{0}$ in Eq. (9). For example, the solution for the case in which the light comes only from the $x<0$ region is expressed as $E_{0}(z)=E_{0}\left(z ; a_{0}^{\prime}\right.$ $=a_{0}, b_{N+1}{ }^{\prime}=0$ ).

As is explained in Appendix $\mathrm{C}$, the required $G\left(z, z^{\prime}\right)$ is given by

$$
\begin{aligned}
G\left(z, z^{\prime}\right) & =\frac{i \tau \exp \left[i k_{N+1}\left(z-z_{N}\right)\right]}{2 k_{0}} E_{0}\left(z^{\prime} ; a_{0}{ }^{\prime}=0, b_{0}^{\prime}\right. \\
& =1) \quad \text { for } z^{\prime}<z, z>z_{N}
\end{aligned}
$$

where $\tau \equiv a_{N+1} / a_{0}$ is the complex transmittance for the unperturbed multilayer. The coefficients $\left\{a_{n}{ }^{\prime}, b_{n}{ }^{\prime}\right\}$ for $E_{0}\left(z^{\prime} ; a_{0}{ }^{\prime}=0, b_{0}{ }^{\prime}=1\right)$ are obtained from Eqs. (C1) and (C2). The final result for $z>z_{N}$ is given as

$$
\begin{aligned}
E(z) \simeq & E_{0}(z)+\frac{i k \tau}{2} \exp \left[i k_{N+1}\left(z-z_{N}\right)\right] \\
& \times\left\{\int _ { - \infty } ^ { 0 } P _ { 1 2 } ^ { ( 0 ) } \eta ( z ^ { \prime } ) \left[a_{0} \exp \left(i k_{0} z^{\prime}\right)\right.\right. \\
& \left.+b_{0} \exp \left(-i k_{0} z^{\prime}\right)\right] \exp \left(-i k_{0} z^{\prime}\right) \mathrm{d} z^{\prime} \\
& +\sum_{n=1}^{N+1} \int_{0}^{d_{n}} P_{12}^{(n)} \eta\left(z^{\prime}+z_{n-1}\right)\left[a_{n} \exp \left(i k_{n} z^{\prime}\right)\right. \\
& \left.+b_{n} \exp \left(-i k_{n} z^{\prime}\right)\right]\left[a_{n}{ }^{\prime} \exp \left(i k_{n} z^{\prime}\right)\right. \\
& \left.+b_{n}{ }^{\prime} \exp \left(-i k_{n} z^{\prime}\right)\right] \mathrm{d} z^{\prime}+\sum_{n=1}^{N+1}\left(a_{n}+b_{n}\right)\left(a_{n}{ }^{\prime}\right. \\
& \left.\left.+b_{n}{ }^{\prime}\right)\left[\epsilon^{(n-1)}-\epsilon^{(n)}\right] u\left(z_{n-1}\right)\right\} .
\end{aligned}
$$

Thus the relative change in the complex transmittance is given by

$$
\begin{aligned}
\frac{\delta \tau}{\tau}= & \frac{i k}{2 a_{0}}\left\{\int _ { - \infty } ^ { 0 } P _ { 1 2 } ^ { ( 0 ) } \eta ( z ^ { \prime } ) \left[a_{0} \exp \left(i k_{0} z^{\prime}\right)\right.\right. \\
& \left.+b_{0} \exp \left(-i k_{0} z^{\prime}\right)\right] \exp \left(-i k_{0} z^{\prime}\right) \mathrm{d} z^{\prime} \\
& +\sum_{n=1}^{N+1} \int_{0}^{d_{n}} P_{12}^{(n)} \eta\left(z^{\prime}+z_{n-1}\right)\left[a_{n} \exp \left(i k_{n} z^{\prime}\right)\right. \\
& \left.+b_{n} \exp \left(-i k_{n} z^{\prime}\right)\right]\left[a_{n}^{\prime} \exp \left(i k_{n} z^{\prime}\right)\right. \\
& \left.+b_{n}{ }^{\prime} \exp \left(-i k_{n} z^{\prime}\right)\right] \mathrm{d} z^{\prime}+\sum_{n=1}^{N+1}\left(a_{n}+b_{n}\right)\left(a_{n}{ }^{\prime}\right. \\
& \left.\left.+b_{n}{ }^{\prime}\right)\left[\epsilon^{(n-1)}-\epsilon^{(n)}\right] u\left(z_{n-1}\right)\right\} .
\end{aligned}
$$

As was done for the reflectance, we describe examples for simple cases. In the case of a single semi-infinite isotropic material whose flat surface is exposed to a vacuum, the change of the transmittance is given as

$$
\begin{aligned}
\frac{\delta \tau}{\tau}= & \frac{i k^{2}}{\left(k+k_{1}\right)}\left\{\int _ { 0 } ^ { z } \Delta \epsilon ( z ^ { \prime } ) \left[\frac{k_{1}-k}{2 k_{1}} \exp \left(2 i k_{1} z^{\prime}\right)\right.\right. \\
& \left.\left.+\frac{k_{1}+k}{2 k_{1}}\right] \mathrm{~d} z^{\prime}+\left(1-\epsilon_{1}\right) u(0)\right\}
\end{aligned}
$$

This can be rewritten as

$$
\begin{aligned}
\frac{\delta \tau}{\tau}= & \frac{i k^{2}}{2 k_{1}} \int_{0}^{z} \Delta \epsilon\left(z^{\prime}\right)\left[\frac{k_{1}-k}{k_{1}+k} \exp \left(2 i k_{1} z^{\prime}\right)+1\right] \mathrm{d} z^{\prime} \\
& +i\left(k-k_{1}\right) u(0)
\end{aligned}
$$

Another example is a layer formed on a substrate. Using the same notation as before, we give the result by 


$$
\begin{aligned}
\frac{\delta \tau}{\tau}= & \frac{i k}{2 a_{0}}\left\{\int_{0}^{d} \Delta \epsilon\left(z^{\prime}\right)\left[a_{1} \exp \left(i k_{1} z^{\prime}\right)+b_{1} \exp \left(-i k_{1} z^{\prime}\right)\right]\right. \\
& \times\left[a_{1}^{\prime} \exp \left(i k_{1} z^{\prime}\right)+b_{1}^{\prime} \exp \left(-i k_{1} z^{\prime}\right)\right] \mathrm{d} z^{\prime} \\
& +\int_{0}^{z} \Delta \epsilon\left(z^{\prime}+d\right) a_{2}\left[a_{2}^{\prime} \exp \left(2 i k_{2} z^{\prime}\right)+b_{2}{ }^{\prime}\right] \mathrm{d} z^{\prime} \\
& +\left(a_{1}+b_{1}\right)\left(a_{1}^{\prime}+b_{1}^{\prime}\right)\left(1-\epsilon_{1}\right) u(0)+a_{2}\left(a_{2}{ }^{\prime}\right. \\
& \left.\left.+b_{2}{ }^{\prime}\right)\left(\epsilon_{1}-\epsilon_{2}\right) u(d)\right\},
\end{aligned}
$$

with

$$
\begin{aligned}
& a_{1}^{\prime}=-\frac{k-k_{1}}{2 k_{1}}, \\
& b_{1}^{\prime}=\frac{k+k_{1}}{2 k_{1}}, \\
& a_{2}^{\prime}=-\frac{\left(k+k_{1}\right)\left(k_{1}-k_{2}\right) \exp \left(-i k_{1} d\right)+\left(k-k_{1}\right)\left(k_{1}+k_{2}\right) \exp \left(i k_{1} d\right)}{4 k_{1} k_{2}}, \\
& b_{2}^{\prime}=\frac{\left(k-k_{1}\right)\left(k_{1}-k_{2}\right) \exp \left(i k_{1} d\right)+\left(k+k_{1}\right)\left(k_{1}+k_{2}\right) \exp \left(-i k_{1} d\right)}{4 k_{1} k_{2}} .
\end{aligned}
$$

The extension of these formulas to the multilayer structure is straightforward. The boundary conditions that must be satisfied are the continuity of $u$ and $\sigma_{z z}$ at each

picosecond time scale when, as we have assumed, the excitation laser spot size is much greater than the multilayer thickness. The second term on the right-hand side of Eq. (22) is the source term. The elastic wave propagates with the longitudinal sound velocity $v$, which is expressed as

$$
\rho v^{2}=3 \frac{1-\nu}{1+\nu} B .
$$

The other coefficients $a_{0}, b_{0}, a_{1}, b_{1}$, and $a_{2}$ were given following Eq. (15).

\section{B. Strain Wave Generation in a Multilayer}

To experimentally test the theoretical predictions, we simulate the strain wave generation, propagation, and detection in a multilayer sample containing metallic and insulating layers. For the strain wave generation, which takes place in the metallic region of the sample, we use a model that involves the following simplifying assumptions: (1) the absorbed pump light pulse sets up an instantaneous temperature rise $\Delta T(z)$; (2) the isotropic thermal stress caused by $\Delta T$ acts as the source term in the strain wave equation; and (3) effects such as the finite optical pulse duration and thermal diffusion are neglected, although the effect of diffusion of nonequilibrium electrons is included in an empirical manner as described below.

The equations of elasticity in one dimension for an elastically isotropic material can be expressed as ${ }^{1}$

$$
\begin{aligned}
\sigma_{z z} & =3 \frac{1-\nu}{1+\nu} B \eta_{z z}-3 B \beta \Delta T(z, t), \\
\rho \frac{\partial^{2} u}{\partial t^{2}} & =\frac{\partial \sigma_{z z}}{\partial z}, \\
u(z, t) & =\int_{+\infty}^{z} \eta_{z z}\left(z^{\prime}, t\right) \mathrm{d} z^{\prime},
\end{aligned}
$$

where $B$ is the bulk modulus, $\nu$ is the Poisson's ratio, $\rho$ is the mass density, $\beta$ is the linear expansion coefficient, and $\sigma_{z z}$ is the longitudinal stress. The other components $\sigma_{x x}$ and $\sigma_{y y}$ do not play an active role in the propagation on a interface and $\sigma_{z z}=0$ at the sample surface. The initial conditions are given by $\eta_{z z}=\dot{\eta}_{z z}=u=0$ at $t=0$.

In the present case, the time dependence of the temperature rise $\Delta T(z, t)$ is given by the step function

$$
\Delta T(z, t)=\Delta T(z) \theta(t) .
$$

This temporal form of the source term makes it possible to include its effect as an initial strain distribution. The procedure to determine the time-varying strain distribution and interface motion is as follows. Using the solution $\eta_{A}, u_{A}$ of the time-independent equations

$$
\begin{aligned}
0 & =\rho v^{2} \eta_{A}-3 B \beta \Delta T(z), \\
u_{A}(z) & =\int_{+\infty}^{z} \eta_{A}\left(z^{\prime}\right) \mathrm{d} z^{\prime},
\end{aligned}
$$

we express the solution $\eta_{z z}(z, t), u(z, t)$ as

$$
\begin{aligned}
\eta_{z z}(z, t) & =\eta_{A}(z)+\eta_{B}(z, t), \\
u(z, t) & =u_{A}(z)+u_{B}(z, t) .
\end{aligned}
$$

Then the elastic wave equation of Eqs. (22)-(24) becomes

$$
\begin{aligned}
\sigma_{z z} & =\rho v^{2} \eta_{B}, \\
\rho \frac{\partial^{2} u_{B}}{\partial t^{2}} & =\frac{\partial \sigma_{z z}}{\partial z}, \\
u_{B}(z, t) & =\int_{+\infty}^{z} \eta_{B}\left(z^{\prime}, t\right) \mathrm{d} z^{\prime},
\end{aligned}
$$

which has the general solution

$$
\eta_{B}(z, t)=f(z-v t)+g(z+v t) .
$$


The initial conditions are given as

$$
\begin{aligned}
& \eta_{B}(z, t=0)=f(z)+g(z)=-\eta_{A}(z), \\
& \dot{\eta}_{B}(z, t=0)=-v\left[\frac{\mathrm{d} f(z)}{\mathrm{d} z}-\frac{\mathrm{d} g(z)}{\mathrm{d} z}\right]=0 .
\end{aligned}
$$

The source term is canceled out in the equation for $\eta_{B}$, and its effect is incorporated into the initial condition. Equations (35) and (36) are sufficient to determine the functions appearing in Eq. (34):

$$
f(z)=g(z)=-\eta_{A}(z) / 2 .
$$

Unique functions $f(z), g(z)$ are defined for each layer in the multilayer sample. To obtain the strain evolution, one should consider the reflection and transmission of the strain wave at the interfaces. We discuss this issue in Section 4.

The temperature rise $\Delta T(z)$ is governed by the heat generated per unit volume $Q(z)$ through light absorption:

$$
\Delta T(z)=Q(z) / C
$$

where $C$ is the specific heat per unit volume. The heat generation rate per unit volume in the $n$th layer $\dot{Q}(z)$ can be calculated from the Poynting vector $\mathbf{S}=\operatorname{Re} \mathbf{E}$ $\times \operatorname{Re} \mathbf{H}$. For normal pump light incidence,

$$
\begin{aligned}
\dot{Q}(z)= & -\operatorname{div} \overline{\mathbf{S}}=\epsilon_{0} \omega N_{n} K_{n}\left[\left|a_{n}\right|^{2} \exp \left(-2 K_{n} k \zeta_{n}\right)\right. \\
& +\left|b_{n}\right|^{2} \exp \left(2 K_{n} k \zeta_{n}\right)+a_{n} b_{n}{ }^{*} \exp \left(2 i N_{n} k \zeta_{n}\right) \\
& \left.+a_{n}{ }^{*} b_{n} \exp \left(-2 i N_{n} k \zeta_{n}\right)\right],
\end{aligned}
$$

where $\overline{\mathbf{S}}$ refers to the time average and, as before, $\zeta_{n}$ $=z-z_{n-1}$. The third and fourth terms in the parentheses arise from the interference between the forwardand backward-propagating light waves. The angular frequency $\omega$ and refractive index $N_{n}+i K_{n}$ here apply to the pump light and likewise for the electric field amplitudes $a_{n}$ and $b_{n}$.

To a first approximation the effect of electron diffusion can be phenomenologically included when we broaden the light penetration depth. ${ }^{12,13}$ This can be done if we introduce the multiplying factor $\mu_{n}(<1)$ for the extinction coefficient $K_{n}$ in the $n$th layer. Then Eq. (39) becomes

$$
\begin{aligned}
\dot{Q}(z)= & \epsilon_{0} \omega \mu_{n} N_{n} K_{n}\left[\left|a_{n}\right|^{2} \exp \left(-2 \mu_{n} K_{n} k \zeta_{n}\right)\right. \\
& +\left|b_{n}\right|^{2} \exp \left(2 \mu_{n} K_{n} k \zeta_{n}\right)+a_{n} b_{n} * \exp \left(2 i N_{n} k \zeta_{n}\right) \\
& \left.+a_{n}{ }^{*} b_{n} \exp \left(-2 i N_{n} k \zeta_{n}\right)\right] .
\end{aligned}
$$

This approximation will break down if diffusing electrons meet an interface. We therefore assume that $d_{n}$ $\gg 1 / k K_{n} \mu_{n}$ in any metallic layer. In the case of no electron diffusion, $\mu=1$.

By integrating Eq. (40) with respect to time, one can obtain an expression for $\eta_{A}(z)$ in the $n$th layer:

$$
\begin{aligned}
\eta_{A n}(z)= & \frac{2 k F \beta_{n} \mu_{n} N_{n} K_{n}}{C_{n}\left|a_{0}\right|^{2}}\left(\frac{1+\nu_{n}}{1-\nu_{n}}\right) \\
& \times\left[\left|a_{n}\right|^{2} \exp \left(-2 \mu_{n} K_{n} k \zeta_{n}\right)\right. \\
& +\left|b_{n}\right|^{2} \exp \left(2 \mu_{n} K_{n} k \zeta_{n}\right) \\
& +a_{n} b_{n} * \exp \left(2 i N_{n} k \zeta_{n}\right) \\
& \left.+a_{n} * b_{n} \exp \left(-2 i N_{n} k \zeta_{n}\right)\right],
\end{aligned}
$$

where $F$ is the incident optical pump pulse fluence (in $\mathrm{Jm}^{-2}$ ) on the front surface of the multilayer and $k$ corresponds here to the vacuum wave vector of the pump light. This can be used to calculate the strain distribution at $t=0$. For other angles of pump incidence and polarization, it is straightforward to generalize Eq. (41).

\section{EXPERIMENT AND RESULTS}

To test the above theory for a nontrivial geometry, but for one in which the number of fitting parameters is not excessive, we carried out an experiment with laser picosecond acoustics on a multilayer sample consisting of a double-layer film made up of transparent and opaque layers on a substrate. A film of vitreous silica $\left(a-\mathrm{SiO}_{2}\right)$ upon a film of polycrystalline chromium was prepared on a fused-silica substrate by rf sputtering at room temperature. Needle profiling techniques gave the thicknesses of the $a-\mathrm{SiO}_{2}$ and $\mathrm{Cr}$ layers as, respectively, 1000 and 100 nm with $10 \%$ accuracy.

The optical pump and probe method is used to generate and detect strain pulses in the multilayer sample. The strain pulses are excited by optical pump pulses at a wavelength of $830 \mathrm{~nm}$ from a mode-locked Ti:sapphire laser and focused to a spot diameter $\sim 20 \mu \mathrm{m}$ on the front surface of the sample at an incident angle of $30^{\circ}$ by use of $s$ polarization. The FWHM (full width at half-maximum) pump pulse duration is $700 \mathrm{fs}$ after passing through an acousto-optic modulator that chops the light at a frequency of $1.5 \mathrm{MHz}$ for lock-in detection purposes. The pulse energy is $1 \mathrm{~nJ}$, and the laser repetition rate is 82 $\mathrm{MHz}$. Second-harmonic light at a wavelength of $415 \mathrm{~nm}$ is used for the probe, with a pulse energy of $0.2 \mathrm{~nJ}$. This produces a transient temperature rise $\sim 4 \mathrm{~K}$ that is small enough for linear theories for the sample response and for the detection process to be appropriate. A delay line provides a variable delay of $0-600 \mathrm{ps}$ between the pump and the probe pulses, the latter focused onto the excitation region at normal incidence also from the front side of the sample. The probe light spot diameter is $\sim 10 \mu \mathrm{m}$.

The presence of the strain pulse causes a timedependent change in the complex reflectance from its initial value $r_{0}=\left|r_{0}\right| \exp \left(i \phi_{0}\right) \quad$ to $\quad r_{0}+\delta r=\left|r_{0}\right|(1$ $+\rho) \exp \left[i\left(\phi_{0}+\delta \phi\right)\right]$, where the real quantities $\rho$ and $\delta \phi$ are the relative change in the modulus of the reflectance and the change in phase, respectively. For the case in which both $\rho$ and $\delta \phi$ are much smaller than unity, $\delta r / r=\rho+i \delta \phi$, and this should be calculable by use of the theoretical result of Eq. (13). In general both $\rho$ and $\delta \phi$ contain contributions from surface or interface displacement as well as from the changes in dielectric constants in the bulk of the sample. Therefore a modified 
Sagnac interferometer geometry ${ }^{4}$ is used here to detect $\rho$ and $\delta \phi$ separately to provide a more stringent test for the theory. The results are shown in Fig. 2. The relative signal intensities in $\rho$ and $\delta \phi$ are retained in the plot by use of the same scale for each (or differing by a factor of 2 ). The typical signal level is $10^{-6} \sim 10^{-5}$.

\section{DISCUSSION}

The main characteristics of the experimental results are as follows: At $t=0$ the optical pump pulse is absorbed in the $\mathrm{Cr}$ substrate, producing a strain, temperature, and carrier distribution localized within a region of the order of the optical skin depth of $\mathrm{Cr}(\sim 15 \mathrm{~nm})$. The strain pulse is transmitted to the $a-\mathrm{SiO}_{2}$ layer, and, for an interval of $\sim 170 \mathrm{ps}$, the outward propagation of the strain pulse within the $a-\mathrm{SiO}_{2}$ layer produces an oscillation in $\rho$ and $\delta \phi$ arising from the interference between the light reflected at the surface or interfaces and at the strain pulse. ${ }^{14}$ The frequency of the oscillation is given by $f$ $=2 N_{1} v_{1} / \lambda \sim 40 \mathrm{GHz}$, where $\lambda$ is the probe wavelength and $N_{1}$ and $v_{1}$ refer to $a-\mathrm{SiO}_{2}$. The change in signal

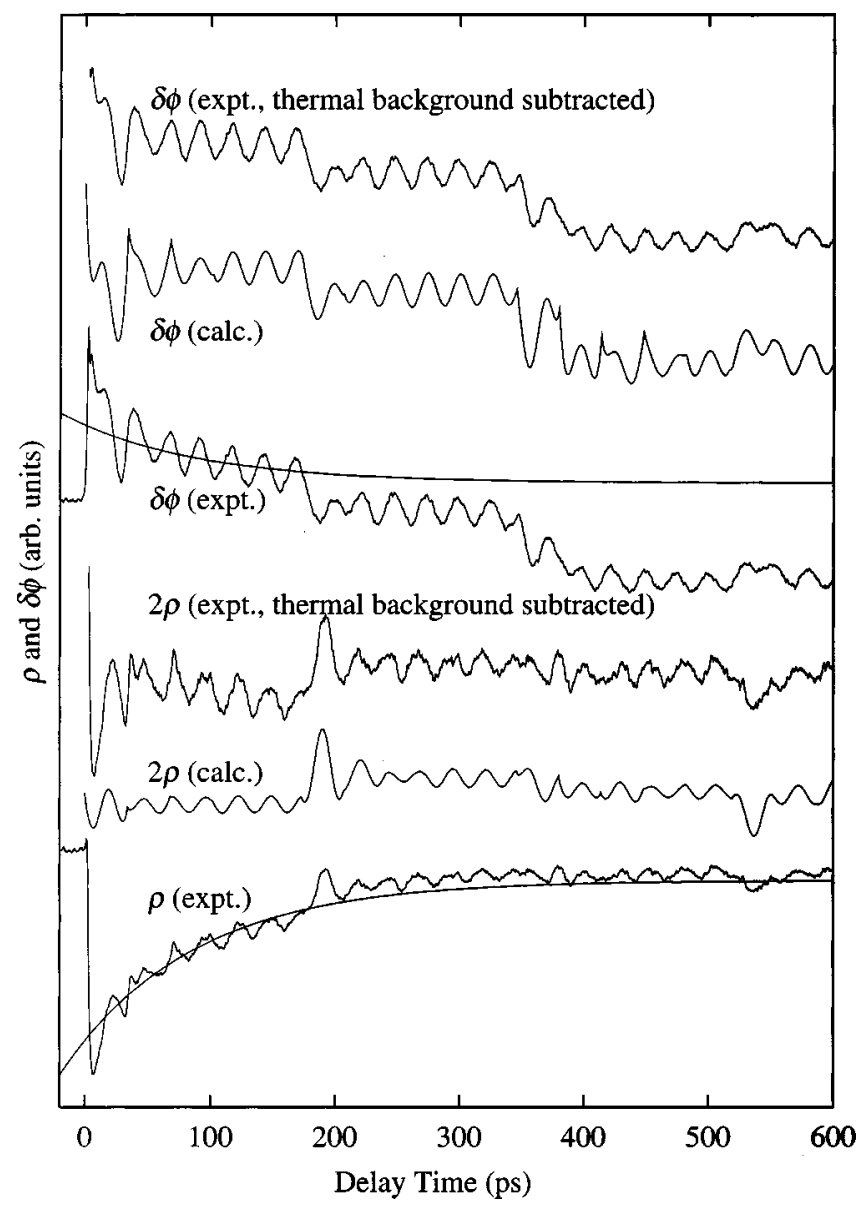

Fig. 2. Change in reflectance $\rho$ (lower solid curve) and phase $\delta \phi$ (upper solid curve) obtained with a $a-\mathrm{SiO}_{2}$-Cr double layer on a fused-silica substrate by use of a modified Sagnac interferometer detection scheme. The fitted thermal background signals (smooth curves) are shown superimposed on the raw data. The theoretical curves are in good agreement with the experimental data after the subtraction of the thermal background. Note that $\rho$ and $\delta \phi$ are plotted on the same vertical scale (or differing by a factor of 2 where indicated). level near $t=0$ is caused by a combination of effects: the motion of the $\mathrm{Cr}-\mathrm{SiO}_{2}$ interface, the change in temperature, and the change in carrier distribution in the $\mathrm{Cr}$. The strain pulse reaches the sample surface at $\sim 170 \mathrm{ps}$ and is reflected with a $\pi$ phase change to produce subsequent signals similar to those in the interval from 0 to $170 \mathrm{ps}$. The abrupt changes in the average signal levels in $\rho$ and $\delta \phi$ at $170 \mathrm{ps}$ are caused by an outward displacement of the sample surface at this time. The magnitude of this displacement is $\sim 1 \mathrm{pm}$.

To analyze the experimental results more precisely, the thermal background of the signal is approximated to a single exponential form with a common time constant for both $\rho$ and $\delta \phi$, and this is subtracted from the raw data (Fig. 2). The resulting curves are then compared with the simulated data calculated by use of the parameters shown in Table 1. The thickness, sound velocities, and photoelastic constants were adjusted as fitting parameters by the least-squares technique. Other parameters are adopted from the literature. The simulation is executed in three stages: the photoinduced strain pulse generation, the strain pulse propagation, and the optical strain pulse detection.

The generation stage is as described in Subsection 2.B, taking full account of the multiple reflection of the pump light. In fact the picosecond strain generation in metals is governed by nonequilibrium electron diffusion and energy transfer between the excited electrons and the lattice. This is well depicted by the two-temperature model describing the evolution of the electron and lattice temperatures. ${ }^{9,11-13}$ In our case, however, the main contribution to the observed signals arises from an integral of the strain over the transparent $\mathrm{SiO}_{2}$ layer, and thus these signals are not sensitive to the detailed strain pulse shape and strain generation mechanisms. We therefore make the simplifying assumption that an instantaneous thermal stress can adequately describe the strain pulse generation. We account for the electronic diffusion in the $\mathrm{Cr}$ layer by setting $\mu=0.5$ for the $\mathrm{Cr}$ layer. This is a reasonable approximation for $\mathrm{Cr}^{2}$ In fact, it turns out that the calculated result here does not depend critically on $\mu$ for $0.5 \leqslant \mu \leqslant 1$. A more precise analysis of the effect of electron diffusion on strain pulse generation in $\mathrm{Cr}$ is given in Ref. 39.

The propagation of the strain wave is traced step by step in the time domain with a sufficiently small time step $\Delta t(=1 \mathrm{ps}$, in this case). Suppose the strain at the time $t=t_{j}$ is expressed as

Table 1. Parameters Used for the Simulation of the Photoacoustic Signals ${ }^{a}$

\begin{tabular}{lcc}
\hline \multicolumn{1}{c}{ Parameter } & $a-\mathrm{SiO}_{2}$ & $\mathrm{Cr}$ \\
\hline Thickness $(\mathrm{nm})$ & 940 & 110 \\
Refractive index at $\lambda=830 \mathrm{~nm}$ & $1.45^{b}$ & $4.26+4.32 i^{c}$ \\
Refractive index at $\lambda=415 \mathrm{~nm}$ & $1.47^{b}$ & $1.54+3.71 i^{c}$ \\
Longitudinal sound velocity $\left(\mathrm{ms}^{-1}\right)$ & 5430 & $6610^{d}$ \\
Mass density $\left(\mathrm{g} \mathrm{cm}^{-3}\right)$ & $2.2^{d}$ & $7.15^{c}$ \\
$\mathrm{~d} N / \mathrm{d} \eta+i \mathrm{~d} K / \mathrm{d} \eta$ & -0.50 & $0.24+0.45 i$ \\
\hline
\end{tabular}

\footnotetext{
${ }^{a}$ Values without a reference number correspond to fitted values

${ }^{b}$ Ref. 36.

${ }^{c}$ Ref. 37.

${ }^{d}$ Ref. 38 .
} 


$$
\eta\left(z, t_{j}\right)=f\left(z-v t_{j}\right)+g\left(z+v t_{j}\right) \equiv F_{j}(z)+G_{j}(z) .
$$

The functions $F_{j}(z)$ and $G_{j}(z)$ correspond to waves propagating in the $+z$ and $-z$ directions, respectively. These components evolve at time $t_{j+1}=t_{j}+\Delta t$ according to

$$
\begin{aligned}
& F_{j+1}(z)=F_{j}(z-v \Delta t), \\
& G_{j+1}(z)=G_{j}(z+v \Delta t),
\end{aligned}
$$

unless $z$ is on an interface. At the interface $z=z_{i}$ between the region $I\left(z<z_{i}\right)$ and $I I\left(z>z_{i}\right)$, the incident strain wave is reflected and transmitted according to the following relations:

$$
\begin{aligned}
F_{j+1}{ }^{I}\left(z_{i}\right)= & F_{j}{ }^{I}\left(z_{i}-v_{I} \Delta t\right), \\
G_{j+1}{ }^{I}\left(z_{i}\right)= & \frac{Z_{I I}-Z_{I}}{Z_{I I}+Z_{I}} F_{j}{ }^{I}\left(z_{i}-v_{I} \Delta t\right) \\
& +\frac{v_{I I}}{v_{I}} \frac{2 Z_{I I}}{Z_{I I}+Z_{I}} G_{j}{ }^{I I}\left(z_{i}+v_{I I} \Delta t\right), \\
F_{j+1}{ }^{I I}\left(z_{i}\right)= & \frac{Z_{I}-Z_{I I}}{Z_{I I}+Z_{I}} G_{j}{ }^{I I}\left(z_{i}+v_{I I} \Delta t\right) \\
& +\frac{v_{I}}{v_{I I}} \frac{2 Z_{I}}{Z_{I I}+Z_{I}} F_{j}{ }^{I}\left(z_{i}-v_{I} \Delta t\right), \\
G_{j+1}{ }^{I I}\left(z_{i}\right)= & G_{j}{ }^{I}\left(z_{i}+v_{I I} \Delta t\right),
\end{aligned}
$$

where $Z=\rho v$ is the acoustic impedance and quantities are labeled $I$ and $I I$ to denote which region they apply to. By combining these relations, we can calculate the strain and thus the displacement for any values of $z$ and $t$.

Once the time evolution of the strain distribution is known, the whole photoacoustic signal can be calculated according to the procedure described in Subsection 2.A, ignoring the contributions from the temperature change and carrier distribution in Eq. (A1). As previously reported, the strain pulses transmitted to the $a-\mathrm{SiO}_{2}$ film are unipolar in shape. ${ }^{14}$ Because the optical and nonequilibrium electron penetration into the $\mathrm{Cr}$ is small compared to the Cr thickness, Eq. (15) can be used to calculate $\delta r / r$. The fitted curves for $\rho$ and $\delta \phi$ are also plotted in Fig. 2, again taking care to use the same scale factor for the predicted $\rho$ and $\delta \phi$ variations to preserve their relative magnitudes. In addition to the detailed signal shape, the relative intensities of $\rho$ and $\delta \phi$ are also well reproduced. One feature of the experimental curves, the slight maximum in $\rho$ (or minimum in $\delta \phi$ ), at $t \sim 170 \mathrm{ps,}$ is also predicted. This is caused by a train of strain pulses (produced by multiple reflection inside the $\mathrm{Cr}$ film) reaching the $a-\mathrm{SiO}_{2}$ surface and producing a temporally staggered response. Moreover, the fitted sound velocities and the photoelastic constant for $a-\mathrm{SiO}_{2}$ (for which comparative data are available) are in reasonable agreement with literature values. ${ }^{14,40,41}$ The accuracies for the fitted values in Table 1 are approximately $\pm 5 \mathrm{~nm}$ for thicknesses, approximately $\pm 25 \mathrm{~ms}^{-1}$ for velocity, and approximately \pm 0.03 for photoelastic constants.

Although similar laser picosecond acoustics experiments have been done on transparent films by use of reflectivity measurements, ${ }^{14,15}$ to our knowledge this re- search represents the first attempt to simultaneously monitor both $\rho$ and $\delta \phi$ for such samples. Therefore this provides a more accurate test of the theory of optical modulation.

We made use of a simplified model for the strain wave generation and propagation. However, the procedures for the calculation of the photoacoustic signal from the spatial perturbation of the optical constants are exactly the same for strain distributions arising from more sophisticated strain generation and propagation models, such as those that would arise with use of the twotemperature model ${ }^{12}$ or with the inclusion of frequencydependent acoustic attenuation. It should also be possible to simulate the raw experimental data when the variations of the dielectric constants with temperature and nonequilibrium electron distributions are included in the model.

\section{SUMMARY}

In conclusion, we have derived general formulas for the reflection and transmission of light incident normally on an arbitrary multilayer structure subject to an inhomogeneous perturbation in its dielectric constants. The formulas can be applied to the calculation of the transient reflectance or transmittance for multilayer structures containing transparent, semitransparent, or opaque layers if one can appropriately model the spatiotemporal modulation of the dielectric constants. The validity of the theory is demonstrated by comparison with experimental transient reflectance and phase signals associated with picosecond strain propagation in a structure containing transparent and opaque layers. From the fitting procedure we have derived some characteristic sample parameters, namely, the thicknesses, sound velocities, and photoelastic constants. This powerful analytical tool should provide a firm foundation for the application of ultrashort pulsed laser techniques to the quantitative investigation of acoustic strain transduction mechanisms, to the analysis of transient temperature and electronic distributions, and to the nondestructive diagnostics and evaluation of complex microstructures and nanostructures in general. The method should also be useful in longer time-scale photoacoustic and photothermal experiments on microstructures.

\section{APPENDIX A: BULK CONTRIBUTION TO THE PERTURBATION OF THE DIELECTRIC TENSOR}

In this appendix we discuss the nature of the bulk contribution to the inhomogeneous modulation of the dielectric constant, that is, $\epsilon_{b}$. We start the discussion from a consideration of the dielectric tensor. The assumed isotropy of the system reduces the complexity of the strain tensor. In addition, because of this isotropy, both the displacement vector connected with the elastic deformation and the propagation direction of the strain wave are parallel to the $z$ axis. The displacement vector $\mathbf{u}$ can therefore be 
expressed as $[0,0, u(z, t)]$, and the only nonzero strain tensor component is $\eta_{z z}(z, t)$.

Inhomogeneous strain, temperature, or carrier distributions can modulate the dielectric tensor; for an optically isotropic material the deviation of the dielectric tensor from its equilibrium value is assumed to be given by ${ }^{42}$

$$
\begin{aligned}
\Delta \epsilon(z, t)= & \eta_{z z}\left[\begin{array}{ccc}
P_{12} & 0 & 0 \\
0 & P_{12} & 0 \\
0 & 0 & P_{11}
\end{array}\right]+(s \Delta T+\gamma \Delta n) \\
& \times\left[\begin{array}{ccc}
1 & 0 & 0 \\
0 & 1 & 0 \\
0 & 0 & 1
\end{array}\right]
\end{aligned}
$$

where $P_{11}, P_{12}, s$, and $\gamma$ are constant coefficients; $\Delta T$ is the temperature deviation from its initial equilibrium value; and $\Delta n$ is the excited carrier density. For the more general case, Eq. (A1) could be extended in principle to include other terms, such as those arising from the electro-optic or the magneto-optic effects. We assume here that the perturbation is small enough to avoid significant second-order contributions in Eq. (A1). There are no off-diagonal components in the dielectric tensor in Eq. (A1). It allows all the components of $\Delta \epsilon_{i j}$ to be determined, but under conditions of normal incidence considered in this paper we in fact are concerned only with $\Delta \epsilon_{x x}$ and $\Delta \epsilon_{y y}$ as described in Subsection 2.A.

The first term in Eq. (A1) associated with the strain arises because of the photoelastic effect. We now take a brief look at the significance of $P_{11}$ and $P_{12}$. Photoelastic tensors are commonly defined through the dielectric impermeability tensor $B_{i j}=1 / \epsilon_{0} \partial E_{i} / \partial D_{j}$ :

$$
\Delta B_{i j}=\pi_{i j k l} \sigma_{k l}=p_{i j r s} \eta_{r s}, \quad p_{i j r s}=\pi_{i j k l} c_{k l r s},
$$

where $\sigma_{k l}$ is the stress, $c_{k l r s}$ is the elastic stiffness, and both $\pi_{i j k l}$ and $p_{i j r s}$ are tensors, like $P_{i j r s}$, that characterize the photoelastic effect. The components of $p_{i j r s}$ are the Pockels coefficients. We define small deviations $\Delta B$ and $\Delta \epsilon$ in $B$ and $\epsilon$. These quantities are related through

$$
(B+\Delta B)_{i j}(\epsilon+\Delta \epsilon)_{j k}=(\epsilon+\Delta \epsilon)_{i j}(B+\Delta B)_{j k}=\delta_{i k} .
$$

Therefore

$$
\begin{aligned}
\Delta \epsilon_{i j} & =-\epsilon_{i \lambda} \Delta B_{\lambda \mu} \epsilon_{\mu j}, \\
\Delta B_{i j} & =-B_{i \lambda} \Delta \epsilon_{\lambda \mu} B_{\mu j} .
\end{aligned}
$$

The relation between the two photoelastic tensors $p_{i j k l}$ and $P_{i j k l}$ can therefore be derived as follows:

$$
\begin{aligned}
\Delta \epsilon_{p q} & =-\epsilon_{p i} \Delta B_{i j} \epsilon_{j q} \\
& =-\epsilon_{p i} \epsilon_{j q} p_{i j r s} \eta_{r s} \\
& \equiv P_{p q r s} \eta_{r s} .
\end{aligned}
$$

So

$$
P_{p q r s}=-\epsilon_{p i} \epsilon_{j q} p_{i j r s} .
$$

In case of an isotropic material, Eq. (A2) is reduced to $P_{p q r s}=-\epsilon^{2} p_{p q r s}$, and the nonzero components of $P_{p q r s}$ are

$$
\begin{aligned}
& P_{11}=P_{22}=P_{33}, \\
& P_{12}=P_{21}=P_{23}=P_{32}=P_{31}=P_{13}, \\
& P_{44}=P_{55}=P_{66}=\frac{1}{2}\left(P_{11}-P_{12}\right),
\end{aligned}
$$

with the abbreviated notation $1=x x, 2=y y, 3=z z$, $4=y z=z y, 5=z x=x z$, and $6=x y=y x$.

In the present treatment we are concerned with the case in which the only nonzero component of $\eta_{i j}$ is $\eta_{z z}$. In this case the components of $\Delta \epsilon_{i j}$ that play a role in optical modulation are

$$
\Delta \epsilon_{x x}=\Delta \epsilon_{y y}=P_{12} \eta_{z z}=-\epsilon^{2} p_{12} \eta_{z z} \equiv \epsilon_{b} .
$$

Expressed in terms of the complex refractive index $\widetilde{n}$ $=\sqrt{\epsilon_{x x}}$,

$$
\Delta \widetilde{n}=\frac{P_{12}}{2 \widetilde{n}} \eta_{z z}=-\frac{\widetilde{n}^{3}}{2} p_{12} \eta_{z z} .
$$

The photoelastic and dielectric constants are complex, wavelength-dependent quantities. Equation (A4) can be rewritten as

$$
\frac{d \widetilde{n}}{\mathrm{~d} \eta}=\frac{\mathrm{d} N}{\mathrm{~d} \eta}+i \frac{\mathrm{d} K}{\mathrm{~d} \eta}=-\frac{\widetilde{n}^{3}}{2} p_{12},
$$

where $\widetilde{n}=N+i K$, and $\eta$ is used as shorthand for $\eta_{z z}$. Similarly, we can write

$$
\frac{\mathrm{d} \epsilon}{\mathrm{d} \eta}=P_{12}=2 \widetilde{n}\left(\frac{\mathrm{d} N}{\mathrm{~d} \eta}+i \frac{\mathrm{d} K}{\mathrm{~d} \eta}\right),
$$

where $\epsilon=\epsilon_{x x}$.

\section{APPENDIX B: INTERFACE CONTRIBUTION TO THE PERTURBATION OF THE DIELECTRIC TENSOR}

In this appendix we consider the interface contribution to the inhomogeneous modulation of the dielectric constants, that is, $\epsilon_{i f}$. The origin of the position coordinate $z$ is defined to be fixed with respect to the equilibrium state of the multilayer. In the presence of elastic deformation, any point of the solid originally at $z$ is displaced to $z+u(z)$. For points in the bulk of the multilayer distant from an interface, there is no need for a linearized theory of the strain to distinguish between the deformed position $z+u(z)$ and the equilibrium position $z .^{43}$ (Such a linearized theory is valid in the present paper for which strain amplitudes are no greater than $\sim 10^{-4}$, implying that $\eta_{z z}$ is related to $u$ through $\eta_{z z}=\partial u / \partial z$.) However, for points in the region of an interface between two media of different dielectric constant, we have to take the displacement of the interface position into account.

This can be seen when we express the perturbed dielectric constant distribution $\epsilon^{\prime}(z)$ in terms of the equilibrium distribution $\epsilon(z)$ :

$$
\begin{aligned}
\epsilon^{\prime}(z) & =\epsilon[z-u(z)]+\Delta \epsilon(z) \\
& =\epsilon[z-u(z)]+P_{12} \eta(z),
\end{aligned}
$$

where we abbreviated $\epsilon_{x x}$ by $\epsilon$ and $\eta_{z z}$ by $\eta$. The presence of $z-u(z)$ in the argument of $\epsilon$ arises because of the movement of material in accordance with the elastic deformation. In the bulk of the sample, in a region dis- 
tant from any interface, we can write $\epsilon[z-u(z)]$ $\simeq \epsilon(z)-(\partial \epsilon / \partial z) u(z)$. The order of magnitude of $\partial \epsilon / \partial z$ and $u(z)$ are, respectively, $\Delta \epsilon / \lambda$ and $\eta \lambda$, where $\eta$ is a typical strain amplitude and $\lambda$ is of the order of the spatial extent of the strain (in practice, of the order of the acoustic wavelength in the case of a propagating strain pulse). The quantity $\lambda$ therefore cancels out, and $\epsilon[z-u(z)]$ $\simeq \epsilon(z)-O(\eta \Delta \epsilon)$. For $\eta \sim 10^{-4}$, the quantity $\epsilon[z$ - $u(z)]$ can therefore be replaced to a good approximation by $\epsilon(z)$ in Eq. (B1) in a region distant from any interface.

In the region of an interface, however, one has to take into account the deformation because, in that case, $\epsilon(z)$ is no longer a good approximation to $\epsilon[z-u(z)]$. As an example, let us consider the spatial dependence of the dielectric constant around the front surface of the multilayer, that is, around the interface at $z=z_{0}$. The dielectric constant in the absence of any inhomogeneous perturbation is given by

$$
\epsilon_{x x}(z)=\left\{\begin{array}{ll}
\epsilon^{(0)} & \text { for } z<z_{0} \\
\epsilon^{(1)} & \text { for } z>z_{0}
\end{array} .\right.
$$

Taking account of the deformation, this is modified to

$$
\epsilon_{x x}{ }^{\prime}(z)=\left\{\begin{array}{ll}
\epsilon^{(0)}+P_{12}^{(0)} \eta(z) & \text { for } z<z_{0}+u\left(z_{0}\right), \\
\epsilon^{(1)}+P_{12}^{(1)} \eta(z) & \text { for } z>z_{0}+u\left(z_{0}\right)
\end{array} .\right.
$$

Equation (B2) represents the effect of the motion of the front surface of the multilayer on $\epsilon_{x x}(z)$ together with that of the photoelastic effect from the bulk. The combined effect from all the interfaces and from the bulk of the sample must be included in the complete analysis.

The inhomogeneous part of the dielectric constant $\epsilon_{i h}$ is conveniently divided into a part because of the bulk contribution $\epsilon_{b}$ and a part because of the movement of the interfaces $\epsilon_{i f}$ :

$$
\epsilon_{i h}=\epsilon_{b}+\epsilon_{i f} \text {. }
$$

According to Eqs. (A1) and (B2), the component $\epsilon_{b}$ in the $n$th layer can be expressed as

$$
\epsilon_{b}(z)=P_{12}^{(n)} \eta(z)
$$

The component $\epsilon_{i f}$ is zero for most values of $z$ except in the small regions encompassing the movement of each interface. Around the interface at $z=z_{n}+u\left(z_{n}\right)$, this component is given by

\section{APPENDIX C: DERIVATION OF THE GREEN'S FUNCTION}

In this appendix we discuss the transfer matrix method to analyze the light-wave propagation in homogeneous multilayers and give the full description of the Green's function.

For the moment we concentrate on finding the relation between the coefficients $a_{n}$ and $b_{n}$ in Eq. (10) according to the boundary conditions at the interfaces, without incorporating the conditions at $z \rightarrow \pm \infty$.

At any of the interfaces of the multilayer, the electric and magnetic field parallel to the interface must be continuous. Because the magnetic field vector is given by

$$
\mathbf{H}(z)=\frac{1}{i \mu_{0} \omega} \operatorname{curl} \mathbf{E}(z),
$$

the boundary condition at $z_{0}$ is expressed as

$$
M_{0}\left[\begin{array}{l}
a_{0} \\
b_{0}
\end{array}\right]=M_{1}\left[\begin{array}{l}
a_{1} \\
b_{1}
\end{array}\right],
$$

and the condition at $z_{n}(n \neq 0)$ as

$$
M_{n} Q_{n}\left[\begin{array}{l}
a_{n} \\
b_{n}
\end{array}\right]=M_{n+1}\left[\begin{array}{l}
a_{n+1} \\
b_{n+1}
\end{array}\right]
$$

where

$$
\begin{gathered}
M_{n} \equiv\left[\begin{array}{cc}
1 & 1 \\
k_{n} & -k_{n}
\end{array}\right], \\
Q_{n} \equiv\left[\begin{array}{cc}
\exp \left(i k_{n} d_{n}\right) & 0 \\
0 & \exp \left(-i k_{n} d_{n}\right)
\end{array}\right] .
\end{gathered}
$$

Because matrices $M_{n}$ and $Q_{n}$ are all regular and have inverse matrices, the equations are reduced to

$$
\left[\begin{array}{l}
a_{0} \\
b_{0}
\end{array}\right]=M\left[\begin{array}{l}
a_{N+1} \\
b_{N+1}
\end{array}\right]
$$

where

$$
M \equiv M_{0}{ }^{-1}\left(\prod_{j=1}^{N} M_{j} Q_{j}^{-1} M_{j}^{-1}\right) M_{N+1} .
$$

Thus if two parameters out of the four, $a_{0}, b_{0}, a_{N+1}$, $b_{N+1}$, are known, then all other coefficients $\left\{a_{n}, b_{n}\right\}$ are uniquely determined.

$\epsilon_{i f}(z)= \begin{cases}{\left[P_{12}^{(n)}-P_{12}^{(n+1)}\right] \eta(z)+\left[\epsilon^{(n)}-\epsilon^{(n+1)}\right]} & \text { for } u\left(z_{n}\right)>0, z_{n}<z<z_{n}+u\left(z_{n}\right), \\ {\left[P_{12}^{(n+1)}-P_{12}^{(n)}\right] \eta(z)+\left[\epsilon^{(n+1)}-\epsilon^{(n)}\right]} & \text { for } u\left(z_{n}\right)<0, z_{n}+u\left(z_{n}\right)<z<z_{n}, \\ 0 & \text { otherwise. }\end{cases}$

As $\epsilon_{i f}$ appears only in the integral of Eq. (9), and the region of the integration is restricted at most to $z_{n}$ $\pm u\left(z_{n}\right)$, the first terms in the nonzero lines of Eq. (B5) produce a second-order effect whereas the second terms produce a first-order effect. Thus we can neglect the first terms in Eq. (B5), giving

$\epsilon_{i f}(z)= \begin{cases}\epsilon^{(n)}-\epsilon^{(n+1)} & \text { for } u\left(z_{n}\right)>0, z_{n}<z<z_{n}+u\left(z_{n}\right) \\ \epsilon^{(n+1)}-\epsilon^{(n)} & \text { for } u\left(z_{n}\right)<0, z_{n}+u\left(z_{n}\right)<z<z_{n} \\ 0 & \text { otherwise. }\end{cases}$ 
Because the light is incident from the $z<0$ side of the multilayer, $b_{N+1}$ can be chosen to be zero because no light is expected to return from the substrate side. In this case $a_{0}$ is the amplitude of the electric field of the incident light. The complex reflectance is then $r=b_{0} / a_{0}$ $=M_{21} / M_{11}$. The complex transmittance is given by $\tau$ $=a_{N+1} / a_{0}=1 / M_{11}$.

Now we proceed to evaluate the Green's function. The right-hand side of Eq. (8) is zero unless $z=z^{\prime}$. The function $G\left(z, z^{\prime}\right)$ therefore can be expressed by two solutions for Eq. (7), corresponding to $z<z^{\prime}$ and $z>z^{\prime}$, that are continuous at $z=z^{\prime}$ :

$$
\lim _{\epsilon \rightarrow+0}[G(z, z-\epsilon)-G(z, z+\epsilon)]=0 .
$$

The above continuity is reasonable because the second derivative in Eq. (8) would otherwise give a singularity other than $\delta\left(z-z^{\prime}\right)$. The function $G\left(z, z^{\prime}\right)$ must also satisfy

$$
\begin{aligned}
-1 & =\lim _{\epsilon \rightarrow+0} \int_{z=z^{\prime}-\epsilon}^{z=z^{\prime}+\epsilon}\left[\frac{\partial^{2}}{\partial z^{2}}+k^{2} \epsilon_{h}(z)\right] G\left(z, z^{\prime}\right) \mathrm{d} z \\
& =\lim _{\epsilon \rightarrow 0}\left[\frac{\partial}{\partial z} G\left(z, z^{\prime}\right)\right]_{z=z^{\prime}-\epsilon}^{z=z^{\prime}+\epsilon} \cdot
\end{aligned}
$$

Because the quantities $E(z)$ and $E_{0}(z)$ in Eq. (9) must contain incident and reflected light components at $z$ $\rightarrow-\infty$ and only transmitted light at $z \rightarrow \infty, G\left(z, z^{\prime}\right)$ must give rise only to a $+z$ propagating component at $z$ $\rightarrow \infty$ and to a $-z$ propagating component at $z \rightarrow-\infty$.

When we use these conditions, it is possible to construct $G\left(z, z^{\prime}\right)$. For this purpose we use the general solution of Eq. (7) that is discussed in Subsection 2.A.2. A possible form of $G\left(z, z^{\prime}\right)$ is then

$$
\begin{aligned}
& G\left(z, z^{\prime}\right) \\
& \quad= \begin{cases}g_{1}\left(z^{\prime}\right) E_{0}\left(z ; a_{0}{ }^{\prime}=0, b_{0}{ }^{\prime}=1\right) & \text { for } z<z^{\prime}, \\
g_{2}\left(z^{\prime}\right) E_{0}\left(z ; a_{0}{ }^{\prime}=1, b_{N+1}{ }^{\prime}=0\right) & \text { for } z>z^{\prime},\end{cases}
\end{aligned}
$$

with $z^{\prime}<0$. The coefficients $g_{1}, g_{2}$ depend only on $z^{\prime}$ and can be determined by the conditions expressed by Eqs. (C4) and (C5). The solution for $G\left(z, z^{\prime}\right)$ for $z>z^{\prime}$ is proportional to that for $E_{0}(z)$ in Eq. (9), where $g_{2}$ is used instead of $a_{0}$.

When measuring the reflectivity, we detect the reflected light far from the sample. It is therefore sufficient to evaluate Eq. (9) at some point $z<0$ where no effect is caused by the strain or other perturbations. This implies that we need to know $G\left(z, z^{\prime}\right)$ at any value of $z^{\prime}$ and at a fixed observation point $z<0$. On the other hand, Eq. (C6) is convenient for expressing $G\left(z, z^{\prime}\right)$ at any value of $z$ and fixed $z^{\prime}$. This dilemma is resolved when we use the reciprocity of the Green's function, which can be demonstrated as follows:

$$
\begin{aligned}
\int G\left(z, z^{\prime \prime}\right)\left[\frac{\partial^{2}}{\partial z^{2}}+k^{2} \epsilon_{h}(z)\right] G\left(z, z^{\prime}\right) \mathrm{d} z \\
-\int G\left(z, z^{\prime}\right)\left[\frac{\partial^{2}}{\partial z^{2}}+k^{2} \epsilon_{h}(z)\right] G\left(z, z^{\prime \prime}\right) \mathrm{d} z \\
=G\left(z^{\prime}, z^{\prime \prime}\right)-G\left(z^{\prime \prime}, z^{\prime}\right)=0,
\end{aligned}
$$

where we calculate the integral by first using Eq. (8) and then by partial integration. Thus we simply obtain the Green's function $G\left(z<0, z^{\prime}\right)$ that we actually need for Eq. (9) by interchanging $z$ and $z^{\prime}$ in $G\left(z, z^{\prime}<0\right)$.

As described above, for the complex reflectance we require only the solution for $G\left(z, z^{\prime}\right)$ for $z^{\prime}<0$. In this case, the functions $g_{1}$ and $g_{2}$ can be readily calculated as

$$
\begin{aligned}
& g_{1}\left(z^{\prime}\right)=\frac{i}{2 k_{0}}\left[\exp \left(i k_{0} z^{\prime}\right)+r \exp \left(-i k_{0} z^{\prime}\right)\right], \\
& g_{2}\left(z^{\prime}\right)=\frac{i}{2 k_{0}} \exp \left(-i k_{0} z^{\prime}\right) .
\end{aligned}
$$

The form for $g_{1}$ has fortuitously the same form as $E_{0}\left(z^{\prime}\right)$ for $z^{\prime}<0$. The result for the Green's function becomes $G\left(z, z^{\prime}\right)$

$$
= \begin{cases}\frac{i}{2 k_{0} a_{0}} \exp \left(-i k_{0} z\right) E_{0}\left(z^{\prime}\right) & \text { for } z<z^{\prime}<0, \\ \frac{i}{2 k_{0} a_{0}} \exp \left(-i k_{0} z^{\prime}\right) E_{0}(z) & \text { for } z>z^{\prime}, z^{\prime}<0 .\end{cases}
$$

The symmetry of this result has a satisfying elegance. The reciprocity gives

$$
\begin{aligned}
& G\left(z, z^{\prime}\right) \\
& = \begin{cases}\frac{i}{2 k_{0} a_{0}} \exp \left(-i k_{0} z^{\prime}\right) E_{0}(z) & \text { for } z^{\prime}<z<0, \\
\frac{i}{2 k_{0} a_{0}} \exp \left(-i k_{0} z\right) E_{0}\left(z^{\prime}\right) & \text { for } z^{\prime}>z, z<0\end{cases}
\end{aligned}
$$

for the required $G\left(z, z^{\prime}\right)$ for Eq. (9)

To evaluate the transmittance, we need the $G\left(z, z^{\prime}\right)$ for $z>z_{N}, z^{\prime}<z$. Using the reciprocity relation of Eq. (C7), we can convert the problem into one for which $G\left(z, z^{\prime}\right)$ is obtained for $z^{\prime}>z_{N}, z<z^{\prime}$. The boundary conditions for $z= \pm \infty$ are exactly the same as those described above. Therefore $G\left(z, z^{\prime}\right)$ is expressed as

$$
\begin{aligned}
& G\left(z, z^{\prime}\right) \\
& \quad= \begin{cases}g_{1}\left(z^{\prime}\right) E_{0}\left(z ; a_{0}{ }^{\prime}=0, b_{0}{ }^{\prime}=1\right) & \text { for } z<z^{\prime}, \\
g_{2}\left(z^{\prime}\right) E_{0}\left(z ; a_{N+1}{ }^{\prime}=1, b_{N+1}{ }^{\prime}=0\right) & \text { for } z>z^{\prime},\end{cases}
\end{aligned}
$$

with $z^{\prime}>z_{N}$. This is essentially the same as Eq. (C6), but the second line is expressed in a different way for later convenience. The functions $g_{1}$ and $g_{2}$ are determined by the conditions of Eqs. (C4) and (C5) as

$$
\begin{aligned}
g_{1}\left(z^{\prime}\right) & =\frac{i \exp \left[i k_{N+1}\left(z^{\prime}-z_{N}\right)\right]}{2 k_{0} M_{11}}, \\
g_{2}\left(z^{\prime}\right) & =\frac{i\left\{M_{11} \exp \left[-i k_{N+1}\left(z^{\prime}-z_{N}\right)\right]-M_{12} \exp \left[i k_{N+1}\left(z^{\prime}-z_{N}\right)\right]\right\}}{2 k_{N+1} M_{11}} .
\end{aligned}
$$


Here, use has been made of the identity

$$
M_{11} M_{22}-M_{12} M_{21}=k_{N+1} / k_{0}
$$

obtained from Eq. (C3). The coefficients $\left\{a_{n}{ }^{\prime}, b_{n}{ }^{\prime}\right\}$ for $E_{0}\left(z^{\prime} ; a_{0}{ }^{\prime}=0, b_{0}{ }^{\prime}=1\right)$ are obtained from Eqs. (C1) and (C2). The reciprocity relation of Eq. (C7) gives the required function as in Eq. (16).

\section{ACKNOWLEDGMENTS}

The authors thank David Hurley for stimulating discussions. This research is partially supported by Grant-inAid for Scientific Research from the Ministry of Education, Science, Sports, and Culture (Japan) and the Murata Science Foundation.

*The e-mail address for O. Matsuda is omatsuda@eng.hokudai.ac.jp.

\section{REFERENCES}

1. C. Thomsen, H. T. Grahn, H. J. Maris, and J. Tauc, "Surface generation and detection of phonons by picosecond light pulses," Phys. Rev. B 34, 4129-4138 (1986).

2. O. B. Wright and K. Kawashima, "Coherent phonon detection from ultrafast surface vibrations," Phys. Rev. Lett. 69, 1668-1671 (1992).

3. B. Perrin, B. Bonello, J. C. Jeannet, and E. Romatet, "Interferometric detection of hypersound waves in modulated structures," Prog. Nat. Sci. Suppl. 6, S444-S448 (1996)

4. D. H. Hurley and O. B. Wright, "Detection of ultrafast phenomena by use of a modified Sagnac interferometer," Opt. Lett. 24, 1305-1307 (1999).

5. W. Chen, Y. Lu, H. J. Maris, and G. Xiao, "Picosecond ultrasonic study of localized phonon surface modes in $\mathrm{Al} / \mathrm{Ag} \mathrm{su}-$ perlattices," Phys. Rev. B 50, 14506-14515 (1994).

6. P. Basséras, S. M. Gracewski, G. W. Wicks, and R. J. D. Miller, "Optical generation of high-frequency acoustic waves in $\mathrm{GaAs} / \mathrm{Al}_{x} \mathrm{Ga}_{1-x} \mathrm{As}$ periodic multilayer structures," J. Appl. Phys. 75, 2761-2767 (1994).

7. A. Bartels, T. Dekorsy, and H. Kurz, "Coherent zone-folded longitudinal acoustic phonons in semiconductor superlattices: excitation and detection," Phys. Rev. Lett. 82, 10441047 (1999).

8. K. Mizoguchi, M. Hase, S. Nakashima, and M. Nakayama, "Observation of coherent folded acoustic phonons propagating in a GaAs/AlAs superlattice by two-color pump-probe spectroscopy," Phys. Rev. B 60, 8262-8266 (1999).

9. H. E. Elsayed-Ali and T. Juhasz, "Femtosecond timeresolved thermomodulation of thin gold films with different crystal structures," Phys. Rev. B 47, 13599-13610 (1993)

10. A. Miklós and A. Lörincz, "Transient thermoreflectance of thin metal films in the picosecond regime," J. Appl. Phys. 63, 2391-2395 (1988)

11. N. D. Fatti, C. Voisin, M. Achermann, S. Tzortzakis, D. Christofilos, and F. Vallée, "Nonequilibrium electron dynamics in noble metals," Phys. Rev. B 61, 16956-16966 (2000).

12. O. B. Wright and V. E. Gusev, "Ultrafast generation of acoustic waves in copper," IEEE Trans. Ultrason. Ferroelectr. Freq. Control 42, 331-338 (1995)

13. O. B. Wright, "Ultrafast nonequilibrium stress generation in gold and silver," Phys. Rev. B 49, 9985-9988 (1994).

14. O. B. Wright, "Thickness and sound velocity measurement in thin transparent films with laser picosecond acoustics," J. Appl. Phys. 71, 1617-1629 (1992).

15. O. B. Wright, "Laser picosecond acoustics in double-layer transparent films," Opt. Lett. 20, 632-634 (1995).

16. V. E. Gusev, "Laser hypersonics in fundamental and applied research,” Acustica Suppl. 82, S37-S45 (1996).
17. J. A. Moon and J. Tauc, "Interference effects in pump-probe spectroscopy of thin films," J. Appl. Phys. 73, 4571-4578 (1993).

18. V. K. Subashiev and A. A. Kukharskii, "The reflection coefficient of optically inhomogeneous solids," Phys. Status Solidi 23, 447-452 (1967).

19. D. E. Aspnes and A. Frova, "Influence of spatially dependent perturbations on modulated reflectance and absorption of solids," Solid State Commun. 7, 155-159 (1969).

20. R. Jacobsson, "Light reflection from films of continuously varying refractive index," in Progress in Optics, E. Wolf, ed. (North-Holland, Amsterdam, 1965), Vol. 5, Chap. 5, pp. 247-286.

21. R. L. Hartman, "Green dyadic calculation for inhomogeneous optical media," J. Opt. Soc. Am. A 17, 1067-1076 (2000).

22. G. Caviglia and A. Morro, "Reflection and transmission of electromagnetic waves in planarly stratified media," Nuovo Cimento B 114, 885-901 (1999).

23. G. Chen and C. L. Tien, "Internal reflection effects on transient photothermal reflectance," J. Appl. Phys. 73, 34613466 (1993).

24. C. A. Paddock and G. L. Eesley, "Transient thermoreflectance from metal films," Opt. Lett. 11, 273-275 (1986).

25. S. D. Brorson, A. Kazeroonian, J. S. Moodera, D. W. Face, T. K. Cheng, E. P. Ippen, M. S. Dresselhaus, and G. Dresselhaus, "Femtosecond room-temperature measurement of the electron-phonon coupling constant $\lambda$ in metallic superconductors," Phys. Rev. Lett. 64, 2172-2175 (1990).

26. T. Q. Qiu and C. L. Tien, "Heat transfer mechanisms during short-pulse laser heating of metals," J. Heat Transfer 115, 835-841 (1993).

27. A. N. Smith, J. L. Hostetler, and P. M. Norris, "Nonequilibrium heating in metal films: an analytical and numerical analysis," Numer. Heat Transfer, Part A 35, 859-873 (1999).

28. J. Shah, Ultrafast Spectroscopy of Semiconductors and Semiconductor Nanostructures (Springer, Heidelberg, Germany, 1996).

29. R. Ziebold, T. Witte, M. Hübner, and R. G. Ulbrich, "Direct observation of Fermi-pressure-driven electron-hole plasma expansion in GaAs on a picosecond time scale," Phys. Rev. B 61, 16610-16618 (2000).

30. H. G. Walther, E. Welsch, and J. Opfermann, "Calculation and measurement of the absorption in multilayer films by means of photoacoustics," Thin Solid Films 142, 27-35 (1986).

31. T. Elperin and G. Rudin, "Thermoelasticity problem for a multilayer coating-substrate assembly irradiated by a laser beam," Int. Commun. Heat Mass Transfer 23, 133-142 (1996).

32. H. Hu, X. Wang, and X. Xu, "Generalized theory of the photoacoustic effect in a multilayer material," J. Appl. Phys. 86, 3953-3958 (1999).

33. J. A. Batista, A. M. Mansanares, E. C. da Silva, C. C. Vaz, and L. C. M. Miranda, "Contrast enhancement in the detection of defects in transparent layered structures: the use of optothermal interference technique in solar cell investigation," J. Appl. Phys. 88, 5079-5086 (2000).

34. F. Abelès, "Optics of thin films," in Advanced Optical Techniques, A. C. S. V. Heel, ed. (Wiley, New York, 1967), Chap. 5, pp. $143-188$

35. M. Born and E. Wolf, Principles of Optics, 7th ed. (Cambridge U. Press, Cambridge, UK, 1999).

36. E. D. Palik, ed., Handbook of Optical Constants of Solids (Academic, Boston, 1998).

37. D. R. Lide, ed., CRC Handbook of Chemistry and Physics, 79th ed. (CRC Press, Boca Raton, Fla., 1998).

38. G. W. C. Kaye and T. H. Laby, Tables of Physical and Chemical Constants, 16th ed. (Longmans, London, 1995).

39. T. Saito, O. Matsuda, and O. B. Wright, "Ultrafast acoustic phonon pulse generation in chromium," Physica B 316317, 304-307 (2002).

40. R. W. Dixon, "Photoelastic properties of selected materials and their relevance for applications to acoustic light modulators and scanners," J. Appl. Phys. 38, 5149-5153 (1967). 
41. O. L. Anderson, "Determination and some uses of isotropic elastic constants of polycrystalline aggregates using singlecrystal data," in Physical Acoustics, W. P. Mason, ed. (Academic, New York, 1965), Vol. 3B, Chap. 2, pp. 43-95.
42. J. F. Nye, Physical Properties of Crystals (Oxford U. Press, Oxford, UK, 1957).

43. B. A. Auld, Acoustic Fields and Waves in Solids, 2nd ed. (Krieger, Malabar, Fla., 1990). 